TRANSACTIONS OF THE

AMERICAN MATHEMATICAL SOCIETY

Volume 351, Number 10, Pages 3953-3974

S 0002-9947(99)02217-5

Article electronically published on March 8, 1999

\title{
MORSE HOMOLOGY FOR GENERATING FUNCTIONS OF LAGRANGIAN SUBMANIFOLDS
}

\author{
DARKO MILINKOVIĆ
}

\begin{abstract}
The purpose of the paper is to give an alternative construction and the proof of the main properties of symplectic invariants developed by Viterbo. Our approach is based on Morse homology theory. This is a step towards relating the "finite dimensional" symplectic invariants constructed via generating functions to the "infinite dimensional" ones constructed via Floer theory in Y.-G. Oh, Symplectic topology as the geometry of action functional. I, J. Diff. Geom. 46 (1997), 499-577.
\end{abstract}

\section{INTRODUCTION}

Let $M$ be a compact smooth manifold and $\pi_{T^{*} M}: T^{*} M \rightarrow M$ its cotangent bundle. Consider the space of paths in $T^{*} M$ starting at the zero section

$$
\Omega:=\left\{\gamma:[0,1] \rightarrow T^{*} M \mid \gamma(0) \in o_{M}\right\}
$$

as a fibration over $M$, given by

$$
\pi_{\Omega}: \Omega \rightarrow M, \pi_{\Omega}(\gamma):=\pi_{T^{*} M}(\gamma(1)) .
$$

For a smooth function (Hamiltonian) $H: T^{*} M \times[0,1] \rightarrow \mathbb{R}$ the classical action functional

$$
\mathcal{A}_{H}: \Omega \rightarrow \mathbb{R}
$$

is defined by

$$
\mathcal{A}_{H}=\int_{\gamma} \theta-\int_{0}^{1} H(\gamma(t), t) d t
$$

where $\theta:=\sum p_{i} d q_{i}$ is the canonical one-form on $T^{*} M$.

If $\xi$ is a vector field along $\gamma \in \Omega$, the first variation of $\mathcal{A}_{H}$ in $\xi$-direction is

$$
d \mathcal{A}_{H}(\gamma) \xi=\int_{0}^{1}\left[\omega\left(\frac{d \gamma}{d t}, \xi\right)-d H(\gamma(t), t) \xi\right] d t+\theta(\xi(1)),
$$

where $\omega:=-d \theta$ is the canonical symplectic form on $T^{*} M$. The fiber derivative of $\mathcal{A}_{H}$ vanishes on the set

$$
\left.\Sigma_{\mathcal{A}_{H}}:=\left\{\gamma \in \Omega \mid \frac{d \gamma}{d t}\right\rfloor \omega=d H(\gamma)\right\}
$$

Received by the editors August 18, 1997.

1991 Mathematics Subject Classification. Primary 58E05; Secondary 57R57, 58F05.

(C)1999 American Mathematical Society 
i.e. on the set of Hamiltonian orbits in $T^{*} M$. If $\phi_{t}^{H}$ is the Hamiltonian isotopy generated by $H$, then it is easy to see that

$$
\phi_{1}^{H}\left(o_{M}\right)=\left\{d \mathcal{A}_{H}(\gamma) \mid \gamma \in \Sigma_{\mathcal{A}_{H}}\right\} .
$$

The action functional (1) can be considered as a special case of the following more general construction. Let $\pi: E \rightarrow M$ be a smooth fibration over $M$ with fibers $F_{e}:=\pi^{-1}(\pi(e))$. Let $S: E \rightarrow \mathbb{R}$ be a smooth function such that $d S(e) \pitchfork \nu^{*} F_{e}$ for any $e \in E$, where $\nu^{*} F_{e}$ is the conormal bundle of $F_{e}$ in $T^{*} E$. Then

$$
\Sigma_{S}:=\left\{e \in E \mid d S(e) \in \nu^{*} F_{e}\right\}
$$

is a smooth submanifold of $E$ of dimension $n=\operatorname{dim} M$. We define a map

$$
i_{S}: \Sigma_{S} \rightarrow T^{*} M
$$

by

$$
i_{S}(e)=\left(T^{*} \pi\right)^{-1}(d S(e)) .
$$

It is easy to prove that the mapping $i_{S}: \Sigma_{S} \rightarrow T^{*} M$ is an exact Lagrangian immersion. Moreover,

$$
i_{S}^{*} \theta=d\left(\left.S\right|_{\Sigma_{S}}\right) .
$$

Definition 1. If $L=i_{S}\left(\Sigma_{S}\right)$, we call $S$ the generating function of an (immersed) Lagrangian submanifold $L \subset T^{*} M$. If $E$ is a vector bundle and $S-Q$ has compact support for some non-degenerate fiberwise quadratic form on $E$, then $S$ is called the generating function quadratic at infinity.

The idea of representing Lagrangian submanifolds through generating functions on a finite dimensional space was used by Hörmander in [9] in calculus of Fourier integral operators. Chaperon $[2,3]$ and Laudenbach and Sikorav [10] in the proof of the Arnold conjecture for Lagrangian intersections in the cotangent bundle used the method of "broken trajectories" of Hamiltonian paths to construct the generating functions for certain Lagrangian submanifolds in $T^{*} M$ as a finite dimensional version of the action functional (1).

Theorem 2 ([10], [16]). If $L \subset T^{*} M$ is an embedded Lagrangian submanifold having a generating function quadratic at infinity and $L_{t}:=\phi_{t}^{H}(L)$ for some Hamiltonian isotopy $\phi_{t}^{H}$, then there exist a vector bundle $E_{1}$ and a family $S_{t}: E \rightarrow \mathbb{R}$ of functions such that $S_{t}$ generates $L_{t}$ for all $t$. In particular, Hamiltonian deformation of zero section admits a generating function quadratic at infinity.

The theory of generating functions was further developed by Sikorav [16], Theret [18], Traynor [19], Viterbo [20] and many other authors. If $S: E \rightarrow \mathbb{R}$ is a generating function for $\mathrm{E} \subset T^{*} M$, then the following modifications of $S$ also generate $L$ :

1. Stabilization $\tilde{S}: E \oplus F \rightarrow \mathbb{R}$ of $S:$

$$
\tilde{S}(q, \zeta, \eta)=S(q, \zeta)+Q(\eta)
$$

for some non-degenerate fiberwise quadratic form $Q$ on $F$.

2. Gauge equivalence:

$$
\tilde{S}=S \circ \Phi
$$

for some fiber-preserving diffeomorphism $\Phi: E \rightarrow E$. 


\section{Addition of a constant:}

$$
\tilde{S}=S+c_{0}
$$

for some constant $c_{0} \in \mathbb{R}$.

The choice of a generating function for a given Lagrangian submanifold $L \subset T^{*} M$ is unique up to these three transformations:

Theorem 3 (Viterbo, [20]). Let $S_{1}$ and $S_{2}$ be two generating functions quadratic at infinity generating the same embedded Lagrangian submanifold $L=\phi_{1}^{H}\left(o_{M}\right)$ in $T^{*} M$. Then, after stabilization, $S_{1}$ is gauge equivalent to $S_{2}+c_{0}$ for some $c_{0} \in \mathbb{R}$.

Note that the after modifications 1.-3. a new $S$ does not have to be quadratic at infinity. However, after a gauge transformation which leaves $S$ unchanged on a prescribed compact set, we can obtain a generating function quadratic at infinity. More precisely, we have the following

Proposition 4 (Proposition 11 [18]). If $Q$ is a fiberwise quadratic form on $E$ and $S: E \rightarrow \mathbb{R}$ is a function such that $\left\|\frac{\partial}{\partial q}(S-Q)\right\|$ is bounded, then for every compact set $K \subset E$ there exists a fiber preserving diffeomorphism $\Phi: E \rightarrow E$ such that $\Phi=i d$ on $K$ and $S \circ \Phi=Q$ at infinity.

In [20] Viterbo developed a rather sophisticated geometric and topological theory of generating functions by studying deformations of their level sets. For set $\mathcal{S}$ of generating functions quadratic at infinity he defined the function

$$
c: H^{*}(M) \times \mathcal{S} \rightarrow \mathbb{R}
$$

and proved that it is essentially independent of the choice of generating function in the class of normalized functions generating a fixed Lagrangian submanifold. Furthermore, he applied this theory to

$$
L:=\operatorname{Graph}(\phi) \subset \mathbb{C}^{n} \times \mathbb{C}^{n}
$$

to construct a biinvariant norm on the Lie group of compactly supported Hamiltonian diffeomorphisms in $\mathbb{C}^{n}$. That construction has a number of interesting implications to the symplectic geometry of $\mathbb{C}^{n}$ : the construction of a symplectic capacity, the construction of a certain biinvariant norm on the group of Hamiltonian diffeomorphisms, a proof of the "Camel Theorem", the existence of infinitely many periodic points of compactly supported symplectic diffeomorphism in the interior of its support and others.

The infinite dimensional analogue of Viterbo's invariants was developed by $\mathrm{Oh}$ [14] by studying the deformation of level sets of the action functional by means of Floer theory. That construction has some applications to Hofer's geometry.

In this paper we will give a construction alternative to Viterbo's, from the point of view of Morse homology. This is a step towards relating the "finite dimensional" symplectic invariants constructed via generating functions to the "infinite dimensional" ones constructed via Floer theory (see [11]).

\section{Morse HOMOLOGY}

We briefly recall the main points of the construction of Morse homology and refer the reader to [15] for a detailed description. The idea of this construction goes back to Smale [17] and Milnor [12] and has been reformulated by Witten [21] and 
Floer [4]-[6]. For a Morse function $f$ on a compact smooth manifold $M$ we denote by $\operatorname{Crit}_{p}(f)$ the set of its critical points of index $p$ and define

$$
C_{p}(f):=\text { free abelian group generated by } \operatorname{Crit}_{p}(f) \text {. }
$$

Consider the gradient flow generated by the equation

$$
\frac{d \gamma}{d t}=-\nabla f
$$

where $\nabla f$ is defined with respect to the given Riemannian metric $g$ on $M$. Denote by $\mathcal{M}_{f, g}(M)$ the set of all $\gamma: \mathbb{R} \rightarrow M$ satisfying (2) such that

$$
\int_{-\infty}^{+\infty}\left|\frac{d \gamma}{d t}\right|^{2} d t<\infty
$$

For a generic choice of a metric, the spaces

$$
\mathcal{M}_{f, g}\left(x^{-}, x^{+}\right)=\left\{\gamma \in \mathcal{M}_{f, g}(M) \mid \gamma(t) \rightarrow x^{ \pm} \text {as } t \rightarrow \pm \infty\right\}
$$

are smooth manifolds of dimension $m\left(x^{+}\right)-m\left(x^{-}\right)$, where $m(x)$ denotes the Morse index of a critical point $x$. Note that

$$
\mathcal{M}_{f, g}(x, y) \cong W_{g}^{u}(x) \cap W_{g}^{s}(y),
$$

where $W_{g}^{s}(y)$ and $W_{g}^{u}(x)$ are the stable and unstable manifolds of the gradient flow (2). For generic $g$ the intersection above is transverse (Morse-Smale condition). The group $\mathbb{R}$ acts on $\mathcal{M}_{f, g}(x, y)$ by $\gamma \mapsto \gamma(\cdot+t)$. We denote

$$
\widehat{\mathcal{M}}_{f, g}(x, y):=\mathcal{M}_{f, g}(x, y) / \mathbb{R} .
$$

The manifolds $\widehat{\mathcal{M}}_{f, g}(x, y)$ can be given a coherent (i.e. compatible with gluing process) orientation $\sigma$ (see [15]).

We define

$$
\begin{gathered}
\partial: C_{p}(f) \rightarrow C_{p-1}(f), \\
\partial x:=\sum_{y \in \operatorname{Crit}_{p-1}(f)} n(x, y) y,
\end{gathered}
$$

where $n(x, y)$ is the number of points in zero dimensional manifold $\widehat{\mathcal{M}}_{f, g}(x, y)$ counted with the sign with respect to the orientation $\sigma$. The proof of $\partial \circ \partial=0$ is based on gluing and cobordism arguments (see, for example, [15]). Now Morse homology groups are defined by

$$
H_{p}^{\mathrm{Morse}}(f):=\operatorname{Ker}(\partial) / \operatorname{Im}(\partial) .
$$

For generic choices of Morse functions $f_{1}$ and $f_{2}$ the groups $H_{p}\left(f_{1}\right)$ and $H_{p}\left(f_{2}\right)$ are isomorphic. Furthermore, they are isomorphic to the singular homology group of $M$, i.e.

$$
H_{p}^{\mathrm{Morse}}(f) \cong H_{p}^{\mathrm{sing}}(M)
$$

for generic $f$. We refer the reader to [12] for the proof.

Wherever there is no possibility of confusion we will omit the superscript Morse in the above notation. Also, we will use the symbol $H_{p}(M)$ to denote the Morse homology defined by means of some Morse function $f: M \rightarrow \mathbb{R}$, wherever it is not necessary to specify $f$. Furthermore, when it is necessary to emphasize the role of a particular Riemannian metric $g$ with respect to which the gradient in 2 is defined, we denote the Morse homology groups by $H_{p}(g, f)$. Finally, when it is necessary to 
emphasize the coherent orientation $\sigma$ used in a definition of a boundary operator $\partial$, we will write $H_{p}^{\sigma}(f)$.

For a later purpose we briefly sketch the construction of isomorphism

$$
h_{\alpha \beta}: H_{p}\left(f^{\alpha}\right) \rightarrow H_{p}\left(f^{\beta}\right)
$$

for generic Morse functions $f^{\alpha}, f^{\beta}$. Consider the "connecting trajectories", i.e. the solutions of non-autonomous equation

$$
\frac{d \gamma}{d t}=-\nabla f_{t}^{\alpha \beta}
$$

where $f_{t}^{\alpha \beta}$ is a homotopy connecting $f^{\alpha}$ and $f^{\beta}$ such that for some $R>0$

$$
\begin{gathered}
f_{t}^{\alpha \beta} \equiv f^{\alpha} \text { for } t \leq-R, \\
f_{t}^{\alpha \beta} \equiv f^{\beta} \text { for } t \geq R .
\end{gathered}
$$

For $x^{\alpha} \in \operatorname{Crit}_{p}\left(f^{\alpha}\right)$ and $x^{\beta} \in \operatorname{Crit}_{p}\left(f^{\beta}\right)$ denote

$$
\mathcal{M}_{f^{\alpha \beta}, g}\left(x^{\alpha}, x^{\beta}\right):=\left\{\gamma \mid \gamma \text { satisfies (4) and } \lim _{t \rightarrow-\infty} \gamma=x^{\alpha}, \lim _{t \rightarrow \infty} \gamma=x^{\beta}\right\} .
$$

As before, $\mathcal{M}_{f^{\alpha \beta}, g}$ is a smooth finite dimensional manifold. Now, define

$$
\left(h_{\alpha \beta}\right)_{\sharp}: C_{p}\left(f^{\alpha}\right) \rightarrow C_{p}\left(f^{\beta}\right)
$$

by

$$
\left(h_{\alpha \beta}\right)_{\sharp} x^{\alpha}=\sum_{x^{\beta} \in \operatorname{Crit}_{p}\left(f^{\beta}\right)} n\left(x^{\alpha}, x^{\beta}\right) x^{\beta}, \text { for } x^{\alpha} \in \operatorname{Crit}_{p}\left(f^{\alpha}\right),
$$

where $n\left(x^{\alpha}, x^{\beta}\right)$ is the algebraic number of points in zero dimensional manifold $\mathcal{M}_{f^{\alpha \beta}, g}\left(x^{\alpha}, x^{\beta}\right)$ counted with the signs defined by the orientation of $\mathcal{M}_{f^{\alpha \beta}, g}$. Homomorphisms $\left(h_{\alpha \beta}\right)_{\sharp}$ commute with $\partial$ and thus define the homomorphisms $h_{\alpha \beta}$ in homology which, in addition, satisfy $h_{\alpha \beta} \circ h_{\beta \gamma}=h_{\alpha \gamma}$. Proof of these facts is similar to the proof of $\partial^{2}=0$ (see [15] for more details).

In the previous construction we fixed the Riemannian metric $g$ on $M$. If we fix a Morse function $f: M \rightarrow \mathbb{R}$ instead, we establish the isomorphism

$$
h_{\alpha \beta}: H_{p}\left(g^{\alpha}, f\right) \rightarrow H_{p}\left(g^{\beta}, f\right)
$$

between the two Morse homology groups defined by means of two generic metrics $g^{\alpha}$ and $g^{\beta}$ in a similar way, by considering the "connecting trajectories",

$$
\frac{d \gamma}{d t}=-\nabla^{g_{t}^{\alpha \beta}} f .
$$

Here $g_{t}^{\alpha \beta}$ is a homotopy connecting $g^{\alpha}$ and $g^{\beta}$ such that for some $R>0$

$$
\begin{gathered}
g_{t}^{\alpha \beta} \equiv g^{\alpha} \text { for } t \leq-R, \\
g_{t}^{\alpha \beta} \equiv g^{\beta} \text { for } t \geq R,
\end{gathered}
$$

and $\nabla^{g}$ is a gradient defined by metric $g$.

Note that $f$ is decreasing along the trajectories solving autonomous gradient equation (2). Therefore, the boundary operator $\partial$ preserves the downward filtration given by level sets of $f$. In other words, if we denote

$$
\operatorname{Crit}_{p}^{\lambda}(f):=\operatorname{Crit}_{p}(f) \cap f^{-1}((-\infty, \lambda])
$$


and

$$
C_{p}^{\lambda}(f):=\text { free abelian group generated by } \operatorname{Crit}_{p}^{\lambda}(f),
$$

then the boundary operator $\partial$ restricts to

$$
\partial^{\lambda}: C_{p}^{\lambda}(f) \rightarrow C_{p-1}^{\lambda}(f) .
$$

Obviously, $\partial^{\lambda} \circ \partial^{\lambda}=0$ and hence we can define the relative Morse homology groups

$$
H_{p}^{\lambda}(f):=\operatorname{Ker}\left(\partial^{\lambda}\right) / \operatorname{Im}\left(\partial^{\lambda}\right)
$$

An obvious inclusion

$$
j^{\lambda}: \operatorname{Crit}_{p}^{\lambda}(f) \rightarrow \operatorname{Crit}_{p}(f)
$$

generates the homomorphism

$$
j_{\sharp}^{\lambda}: C_{p}^{\lambda}(f) \rightarrow C_{p}(f)
$$

which commutes with $\partial$, i.e. $\partial^{\lambda} \circ j_{\sharp}^{\lambda}=j_{\sharp}^{\lambda} \circ \partial$. Hence, we have an inclusion homomorphism

$$
j_{*}^{\lambda}: H_{p}^{\lambda}(f) \rightarrow H_{p}(f)
$$

Following the standard algebraic construction, we define (relative) Morse cohomology. We set

$$
C_{\lambda}^{p}(f):=\operatorname{Hom}\left(C_{p}^{\lambda}(f), \mathbb{Z}\right)
$$

and

$$
\delta^{\lambda}: C_{\lambda}^{p}(f) \rightarrow C_{\lambda}^{p+1}(f), \quad\left\langle\delta^{\lambda} a, x\right\rangle:=\left\langle a, \partial^{\lambda} x\right\rangle
$$

and define

$$
H_{\lambda}^{p}(f):=\operatorname{Ker}\left(\delta^{\lambda}\right) / \operatorname{Im}\left(\delta^{\lambda}\right) .
$$

Since $\operatorname{Crit}_{p}(f)$ is finite, for large $\lambda$ we have $H_{p}^{\lambda}(f)=H_{p}(f)$ and $H_{\lambda}^{p}(f)=H^{p}(f)$. Homomorphism $j_{\sharp}^{\lambda}$ yields

$$
j_{\lambda}^{\sharp} C^{p}(f) \rightarrow C_{\lambda}^{p}(f), \quad\left\langle j_{\lambda}^{\sharp} a, x\right\rangle:=\left\langle a, j_{\sharp}^{\lambda} x\right\rangle .
$$

Hence, we have the restriction homomorphism

$$
j_{\lambda}^{*}: H^{p}(f) \rightarrow H_{\lambda}^{p}(f) .
$$

Definition of Morse homology and cohomology with coefficients in arbitrary ring $R$ is straightforward.

\section{Poincaré Duality and Cohomology operations}

3.1. Poincaré duality. Let $R$ be a ring and let $M$ be a compact $R$-oriented smooth manifold of dimension $n$. We will assume that all homology and cohomology groups in this section are with coefficients in $R$. Let $f: M \rightarrow \mathbb{R}$ be a Morse function. The obvious bijection

$$
\mathrm{Crit}_{p}(-f) \rightarrow \mathrm{Crit}_{n-p}(f), x \mapsto x
$$

gives rise to an isomorphism

$$
\begin{gathered}
\Gamma: C_{p}(-f) \rightarrow C^{n-p}(f), \\
\Gamma x=\delta_{x}, \text { for } x \in \operatorname{Crit}_{p}(-f) .
\end{gathered}
$$


The correspondence

$$
\Psi: \gamma \mapsto \tilde{\gamma}, \quad \tilde{\gamma}(t):=\gamma(-t)
$$

gives rise to the diffeomorphism

$$
\Psi: \mathcal{M}_{f, g}(y, x) \stackrel{\cong}{\rightrightarrows} \mathcal{M}_{-f, g}(x, y) .
$$

Let $\sigma$ be a canonical coherent orientation of $\mathcal{M}_{f, g}(M)$ and let $\tilde{\sigma}$ denote a pushforward of $\sigma$ to $\mathcal{M}_{-f, g}(M)$ by $\Psi$. Then

$$
n_{\tilde{\sigma}}(x, y)=n_{\sigma}(y, x)
$$

where $n_{\tilde{\sigma}(x, y)}$ (respectively $\left.n_{\sigma}(y, x)\right)$ is the number of points in the zero dimensional manifold $\mathcal{M}_{-f, g}(x, y)$ (respectively $\mathcal{M}_{f, g}(y, x)$ ) counted with respect to the orientation $\tilde{\sigma}$ (respectively $\sigma$ ). Let $\partial_{\tilde{\sigma}}$ and $\delta^{\sigma}$ be the boundary and coboundary operators defined with respect to the orientations $\sigma$ and $\tilde{\sigma}$. Then

$$
\begin{aligned}
\left\langle\delta^{\sigma} \circ \Gamma(x), y\right\rangle & =\left\langle\Gamma(x), \partial_{\sigma} y\right\rangle \\
& =\sum_{z} n_{\sigma}(y, z)\left\langle\delta_{x}, z\right\rangle \\
& =n_{\sigma}(y, x)
\end{aligned}
$$

and

$$
\begin{aligned}
\left\langle\Gamma \circ \partial_{\tilde{\sigma}}(x), y\right\rangle & =\left\langle\delta_{\partial_{\tilde{\sigma}}(x)}, y\right\rangle \\
& =\sum_{z} n_{\tilde{\sigma}}(x, z)\left\langle\delta_{z}, y\right\rangle \\
& =n_{\tilde{\sigma}}(x, y) .
\end{aligned}
$$

Together with (6) this proves

$$
\Gamma \circ \partial_{\tilde{\sigma}}=\delta^{\sigma} \circ \Gamma
$$

Therefore, we have the isomorphism

$$
\Gamma_{*}: H_{p}^{\tilde{\sigma}}(-f) \rightarrow H_{\sigma}^{n-p}(f) .
$$

Denote by

$$
h_{ \pm}: H_{p}^{\tilde{\sigma}}(f) \rightarrow H_{p}^{\tilde{\sigma}}(-f)
$$

the isomorphism (3). Note that, in general, $H_{p}^{\sigma}(f) \neq H_{p}^{\tilde{\sigma}}(f)$ (see Example 4.1.4 [15] or [13]). However, for oriented $M, \partial_{\sigma}=(-1)^{p+1} \partial_{\tilde{\sigma}}$ (see Theorem 7.5 [12]) and hence the isomorphism

$$
C_{p}(f) \rightarrow C_{p}(f), x \mapsto x
$$

gives rise to the isomorphism

$$
\tau_{f}: H_{p}^{\sigma}(f) \stackrel{\cong}{\rightrightarrows} H_{p}^{\tilde{\sigma}}(f) .
$$

This gives rise to Poincaré duality isomorphism

$$
\mathrm{PD}:=\Gamma_{*} \circ h_{ \pm} \circ \tau_{f}: H_{p}^{\sigma}(f) \rightarrow H_{\sigma}^{n-p}(f) .
$$


3.2. Cup product. The classical cup product

$$
\cup: H^{p}(M ; R) \otimes H^{q}(M ; R) \rightarrow H^{p+q}(M ; R)
$$

can be defined in Morse cohomology, by considering the graph moduli spaces associated to generic triple $\left(f_{1}, f_{2}, f_{3}\right)$ of Morse functions on $M$. For a later purpose, we only sketch the main points of the construction, referring the reader to $[1,7,8,13]$ for more details. For a given triple of critical points

$$
\left(x_{1}, x_{2}, x_{3}\right) \in \operatorname{Crit}\left(f_{1}\right) \times \operatorname{Crit}\left(f_{2}\right) \times \operatorname{Crit}\left(-f_{3}\right)
$$

we consider the set $\mathcal{M}\left(f_{1}, f_{2}, f_{3} ; x_{1}, x_{2}, x_{3}\right)$ of paths $\gamma:=\left(\gamma_{1}, \gamma_{2}, \gamma_{3}\right)$,

$$
\begin{gathered}
\gamma_{i}:[0, \infty) \rightarrow M, \quad i=1,2, \\
\gamma_{3}(-\infty, 0] \rightarrow M
\end{gathered}
$$

which satisfy

$$
\left\{\begin{array}{l}
\frac{d \gamma_{i}}{d t}=-\nabla f_{i}\left(\gamma_{i}\right), \\
\lim _{t \rightarrow+\infty} \gamma_{1}(t)=x_{1}, \\
\lim _{t \rightarrow+\infty} \gamma_{2}(t)=x_{2}, \\
\lim _{t \rightarrow-\infty} \gamma_{3}(t)=x_{3}, \\
\gamma_{1}(0)=\gamma_{2}(0)=\gamma_{3}(0) .
\end{array}\right.
$$

For generic choice of parameters the set $\mathcal{M}\left(f_{1}, f_{2}, f_{3} ; x_{1}, x_{2}, x_{3}\right)$ is a smooth manifold of dimension $m\left(x_{3}\right)-m\left(x_{2}\right)-m\left(x_{1}\right)$. When $m\left(x_{3}\right)=m\left(x_{1}\right)+m\left(x_{2}\right)$, the zero dimensional manifold $\mathcal{M}\left(f_{1}, f_{2}, f_{3} ; x_{1}, x_{2}, x_{3}\right)$ is compact. We denote the algebraic number of its points by $n\left(x_{1}, x_{2}, x_{3}\right)$ and define

$$
\Psi\left(f_{1}, f_{2}, f_{3}\right):=\sum_{x_{1}, x_{2}, x_{3}} n\left(x_{1}, x_{2}, x_{3}\right) x_{1} \otimes x_{2} \otimes x_{3} \in C_{*}\left(f_{1}\right) \otimes C_{*}\left(f_{2}\right) \otimes C_{*}\left(-f_{3}\right) .
$$

We define

$$
\cup: H^{p}\left(f_{1}\right) \otimes H^{q}\left(f_{2}\right) \rightarrow H^{p+q}\left(f_{3}\right)
$$

in the following way. For given $a_{1} \in H^{p}\left(f_{1}\right)$ and $a_{2} \in H^{q}\left(f_{2}\right)$ we choose their representative cycles, denoted again by $a_{1}$ and $a_{2}$. Consider the contraction cycle

$$
\begin{aligned}
\left.a_{1} \otimes a_{2}\right\rfloor \Psi & :=\left\langle a_{1} \otimes a_{2}, \Psi\left(f_{1}, f_{2}, f_{3}\right)\right\rangle \\
& \in C_{n-(p+q)}\left(-f_{3}\right)
\end{aligned}
$$

and its dual cocycle

$$
\left.\Gamma\left(a_{1} \otimes a_{2}\right\rfloor \Psi\right) \in C^{p+q}\left(f_{3}\right) .
$$

Now $a_{1} \cup a_{2}$ is defined to be a cohomology class of $\left.\Gamma\left(a_{1} \otimes a_{2}\right\rfloor \Psi\right)$.

If

$$
h_{i}^{\alpha \beta}: H^{*}\left(f_{i}^{\alpha}\right) \rightarrow H^{*}\left(f_{i}^{\beta}\right) \text { for } i \in\{1,2,3\}
$$

is the isomorphism dual to (3), then

$$
h_{3}^{\alpha \beta}\left(a_{1} \cup a_{2}\right)=h_{1}^{\alpha \beta}\left(a_{1}\right) \cup h_{2}^{\alpha \beta}\left(a_{2}\right) .
$$


3.3. Cap product. Let $M$ be a smooth compact $n$-manifold. Assume again that $M$ is $R$-oriented of some ring $R$; Morse homology and cohomology groups will be again with coefficients in $R$. Let $A \subset M$ be a closed $R$-oriented $q$-dimensional submanifold and let

$$
a:=[A] \in H_{q}(M ; R) \text { and } P D(a) \in H^{n-q}(M ; R)
$$

be its singular homology class and its Poincaré dual. We denote

$$
\mathcal{M}_{f, g}^{A}(x, y):=\left\{\gamma \in \mathcal{M}_{f, g}(x, y) \mid \gamma(0) \in A\right\},
$$

where $x, y$ are the critical points of $f$ such that $\operatorname{dim} \mathcal{M}_{f, g}(x, y)=n-q$. By transversality, $\mathcal{M}_{f, g}^{A}(x, y)$ is an oriented zero dimensional manifold for generic choice of $A$.

By a standard compactness result in Morse theory (see for example [15]), any sequence $\gamma_{k} \in \mathcal{M}_{f, g}^{A}(x, y)$ has a subsequence converging to a family

$$
\left(\gamma^{1}, \ldots, \gamma^{s}\right) \in \mathcal{M}_{f, g}\left(x, x^{1}\right) \times \cdots \times \mathcal{M}_{f, g}\left(x^{i}, x^{i+1}\right) \cdots \times \mathcal{M}\left(x^{s-1}, y\right),
$$

with

$$
m(x)<m\left(x^{1}\right)<\cdots<m\left(x^{s-1}\right)<m(y)
$$

where $m$ denotes the Morse index. Moreover, since $\gamma_{k}(0) \in A$, in a neighborhood of $0 \in \mathbb{R} \gamma_{k}$ converges to some $\gamma \in \mathcal{M}_{f, g}^{A}$, which means that

$$
\gamma^{j} \in \mathcal{M}_{f, g}^{A}\left(x^{j}, x^{j+1}\right) \text { for some } j .
$$

By (8) if $s>0$, then

$$
m\left(x^{j}\right)-m\left(x^{j+1}\right)<n
$$

and hence $\operatorname{dim} \mathcal{M}_{f, g}^{A}\left(x^{j}, x^{j+1}\right)<0$, which contradicts (9). Therefore $s=0$, which means that some subsequence of $\gamma_{k}$ converges in $\mathcal{M}_{f, g}^{A}(x, y)$. Hence $\mathcal{M}_{f, g}^{A}(x, y)$ is compact, i.e. finite.

Denote by $n_{A}(x, y)$ the algebraic number of points in $\mathcal{M}_{f, g}^{A}(x, y)$. Let

$$
\psi_{A}: C_{n-q+r}(f) \rightarrow C_{r}(f)
$$

be a map generated by

$$
x \mapsto \sum_{y \in \operatorname{Crit}_{r}(f)} n_{A}(x, y) y, \text { for } x \in \operatorname{Crit}_{n-q+r}(f) .
$$

Note that

$$
\begin{aligned}
\partial \circ \psi_{A}(x) & =\partial\left(\sum_{y} n_{A}(x, y) y\right) \\
& =\sum_{z} \sum_{y} n_{A}(x, y) n(y, z) z
\end{aligned}
$$

and

$$
\begin{aligned}
\psi_{A} \circ \partial(x) & =\psi_{A}\left(\sum_{y} n(x, y) y\right) \\
& =\sum_{z} \sum_{y} n(x, y) n_{A}(y, z) z .
\end{aligned}
$$


The numbers $n_{A}(x, y) n(y, z)$ and $n(x, y) n_{A}(y, z)$ are the algebraic numbers of points in

$$
\mathcal{M}_{f, g}^{A}(x, y) \times \mathcal{M}_{f, g}(x, z) \text { and } \mathcal{M}_{f, g}(x, y) \times \mathcal{M}_{f, g}^{A}(x, z) .
$$

From compactness and gluing arguments (see $[8,15]$ for analytic details) it follows that the points in (12) are precisely the boundary points of the one dimensional manifold $\mathcal{M}_{f, g}^{A}(x, z)$ and hence they appear in pairs with the opposite orientation. Therefore, from (10) and (11) we deduce that $\psi_{A}$ is a chain map, which gives rise to the map in homology

$$
\left(\psi_{A}\right)_{*}: H_{n-q+r}(f) \rightarrow H_{r}(f) .
$$

By similar gluing and compactness arguments we conclude that, if

$$
h_{\alpha \beta}: H_{*}\left(f^{\alpha}\right) \rightarrow H_{*}\left(f^{\beta}\right)
$$

is the isomorphism (3), then

$$
h_{\alpha \beta} \circ\left(\psi_{A}\right)_{*}=\left(\psi_{A}\right)_{*} \circ h_{\alpha \beta}(x) .
$$

Example 5. Let us consider the special case $q=0$. Take the generator $a \in$ $H_{0}(M ; R)$. The cycle representing $a$ is a point $p \in M$, and $P D(a)$ is the volume class $\mu_{M} \in H^{n}(M ; R)$. For generic choice of $p \in M$ the unique solution $\gamma$ of

$$
\frac{d \gamma}{d t}+\nabla^{g} f(\gamma)=0, \gamma(0)=p
$$

defines the map

$$
\begin{gathered}
\operatorname{Crit}_{n}(f) \rightarrow \operatorname{Crit}_{0}(f), \\
x \mapsto \begin{cases}\gamma(+\infty) & \text { if } x=\gamma(-\infty), \\
0 & \text { otherwise }\end{cases}
\end{gathered}
$$

which gives rise to $\psi_{p}$. In this case the map

$$
\left(\psi_{p}\right)_{*}: H_{n}(f) \rightarrow H_{0}(f)
$$

is an isomorphism and

$$
\left(\psi_{p}\right)_{*}=\mu_{M} \cap .
$$

(see Proposition 2.6 [5] or Example 2 [1]).

3.4. Cross product. Another classical product in (co)homology, the cross product, is also defined in Morse category. We sketch the main points of a definition, referring the reader to [15] for details. Let $M_{1}$ and $M_{2}$ be smooth compact manifolds and

$$
f_{1}: M_{1} \rightarrow \mathbb{R}, f_{2}: M_{2} \rightarrow \mathbb{R}
$$

Morse functions. Then

$$
\begin{gathered}
f_{1} \oplus f_{2}: M_{1} \times M_{2} \rightarrow \mathbb{R}, \\
f_{1} \oplus f_{2}(x, y):=f_{1}(x)+f_{2}(y)
\end{gathered}
$$

defines a Morse function on $M \times N$. The set of critical points of $f_{1} \oplus f_{2}$ of Morse index $p$ is

$$
\operatorname{Crit}_{p}\left(f_{1} \oplus f_{2}\right)=\bigcup_{p_{1}+p_{2}=p} \operatorname{Crit}_{p_{1}}\left(f_{1}\right) \times \operatorname{Crit}_{p_{2}}\left(f_{2}\right) .
$$


The assignment

$$
\times: C_{p_{1}}\left(f_{1}\right) \otimes C_{p_{2}}\left(f_{2}\right) \rightarrow C_{p_{1}+p_{2}}\left(f_{1} \oplus f_{2}\right)
$$

defined by

$$
x_{1} \otimes x_{2} \stackrel{\times}{\longmapsto}\left(x_{1}, x_{2}\right), \text { for } x_{i} \in \operatorname{Crit}_{p_{i}}\left(f_{i}\right),
$$

is a chain isomorphism which gives rise to the operation

$$
\times: H_{p_{1}}\left(f_{1}\right) \otimes H_{p_{2}}\left(f_{2}\right) \rightarrow H_{p_{1}+p_{2}}\left(f_{1} \oplus f_{2}\right) .
$$

By duality, the assignment

$$
\times: C^{p_{1}}\left(f_{1}\right) \otimes C^{p_{2}}\left(f_{2}\right) \rightarrow C^{p_{1}+p_{2}}\left(f_{1} \oplus f_{2}\right)
$$

defined by

$$
\langle a \times b, u \times v\rangle:=\langle a, u\rangle\langle b, v\rangle
$$

gives rise to the operation

$$
\times: H^{p_{1}}\left(f_{1}\right) \otimes H^{p_{2}}\left(f_{2}\right) \rightarrow H^{p_{1}+p_{2}}\left(f_{1} \oplus f_{2}\right) .
$$

\section{Viterbo's inVARIANTS}

Let $Q$ be a fixed quadratic function of index $k$ on $\mathbb{R}^{m}$ and $g_{0}$ a standard Euclidean metric on $R^{m}$. Fix a relatively compact open set $K \subset E:=M \times \mathbb{R}^{m}$ and Riemannian metric $g_{M}$ on $M$ and denote

$$
\mathcal{S}_{(E, Q)}:=\{S: E \rightarrow \mathbb{R} \mid S(x, \xi) \equiv Q(\xi) \text { outside } K\}
$$

and

$\mathcal{G}_{g_{M} \oplus g_{0}}:=$ the set of metrics on $E$ which coincide with $g_{M} \oplus g_{0}$ outside $K$.

Morse theory applies for parameters in $\mathcal{S}_{(E, Q)} \times \mathcal{G}_{g_{M} \oplus g_{0}}$ [15]. Let $i: N \hookrightarrow M$ be a smooth closed submanifold. Denote by $S_{N}$ the restriction of $S \in \mathcal{S}_{(E, Q)}$ to $i^{*} E$. Since $S=Q$ outside $K$, the set of critical points of $S_{N}$ is finite. Furthermore, for a family $S_{t}^{\alpha \beta} \in \mathcal{S}_{(E, Q)}$ and for $g \in \mathcal{G}_{g_{M} \oplus g_{0}}$ the solutions of an equation

which satisfy

$$
\frac{d \gamma}{d t}+\nabla^{g}\left(S_{t}^{\alpha \beta}\right)_{N}(\gamma)=0
$$

$$
\int_{-\infty}^{+\infty}\left|\frac{d \gamma}{d t}\right|^{2} d t<\infty
$$

remain in $K$ (note that all $S_{t}^{\alpha \beta}$ coincide with the same fixed $Q$ outside $K$ ). Therefore, the construction of Morse homology groups $H_{*}\left(S_{N}\right)$ is the same as in a compact case.

For a Morse function $f: N \rightarrow \mathbb{R}$ we have obvious bijections

$$
\varphi: \operatorname{Crit}(f) \stackrel{\cong}{\rightrightarrows} \operatorname{Crit}(f \oplus Q), x \mapsto(x, 0)
$$

and

$$
\mathcal{M}_{f, g_{M}} \cong \mathcal{M}_{f \oplus Q, g_{M} \oplus g_{0}}, \gamma \mapsto(\gamma, 0)
$$

This gives rise to the chain isomorphism

$$
\phi_{p}: C_{p}(f) \rightarrow C_{p+k}(f \oplus Q), \phi_{p}\left(\sum x_{i}\right):=\sum \varphi\left(x_{i}\right)
$$


and the cochain isomorphism

$$
\phi^{p}: C^{p}(f) \rightarrow C^{p+k}(f \oplus Q),\left\langle\phi_{p} x, \phi^{p} y\right\rangle:=\langle x, y\rangle .
$$

Hence, we have the isomorphisms

$$
\Phi_{p}: H_{p}(f) \rightarrow H_{p+k}(f \oplus Q) \text { and } \Phi^{p}: H^{p}(f) \rightarrow H^{p+k}(f \oplus Q) .
$$

Remark 6. Function $f \oplus Q$ does not belong to the space $\mathcal{S}_{(E, Q)}$. However, after composing $f \oplus Q$ with a fiber preserving diffeomorphism as in Proposition 4 we obtain the function in $\mathcal{S}_{(E, Q)}$. Since the gauge transformation does not change the Morse complex with suitably chosen metric (see the proof of Lemma 9 below for more details), we will denote the homology and cohomology groups of the new function again by $H_{p}(f \oplus Q)$ and $H^{p}(f \oplus Q)$. The same remark applies for any stabilization $S \sim S \oplus Q$.

By composing $\Phi$ with the connecting isomorphisms

$$
h_{f S}: H_{p+k}(f \oplus Q) \rightarrow H_{p+k}\left(S_{N}\right)
$$

and

$$
h^{f S}: H^{p+k}(f \oplus Q) \rightarrow H^{p+k}\left(S_{N}\right)
$$

we obtain the isomorphisms

$$
T_{p}: H_{p}(N) \rightarrow H_{p+k}\left(S_{N}\right), T_{p}:=h_{f S} \circ \Phi_{p}
$$

and

$$
T^{p}: H^{p}(N) \rightarrow H^{p+k}\left(S_{N}\right), T^{p}:=h^{f S} \circ \Phi^{p} .
$$

Note that if

$$
T_{*}^{\alpha}: H_{*}(f) \rightarrow H_{*}\left(S^{\alpha}\right), T_{*}^{\beta}: H_{*}(f) \rightarrow H_{*}\left(S^{\beta}\right),
$$

or, equivalently,

$$
T_{*}^{\alpha}: H_{*}(f) \rightarrow H_{*}\left(g^{\alpha}, S\right), T_{*}^{\beta}: H_{*}(f) \rightarrow H_{*}\left(g^{\beta}, S\right),
$$

then $h_{\alpha \beta} T_{*}^{\alpha}=T_{*}^{\beta}$.

Definition 7. Let $N \subset M$ be a closed smooth submanifold. Fix $S \in \mathcal{S}_{(E, Q)}$ such that the restriction $S_{N}$ is a Morse function.

1. For $a \in H_{*}(N)$ we define

$$
c(a, S: N):=\inf \left\{\lambda \mid T_{*} a \in \operatorname{Im}\left(j_{*}^{\lambda}\right) \subset H_{*}^{\lambda}\left(S_{N}\right)\right\} .
$$

2. For $u \in H^{*}(N)$ we define

$$
c(u, S: N):=\inf \left\{\lambda \mid j_{\lambda}^{*} T^{*} u \neq 0 \in H_{\lambda}^{*}\left(S_{N}\right)\right\} .
$$

In particular, when $N=M$ we write simply $c(a, S)$ and $c(u, S)$.

Although in a definition of Morse homology (and hence of $c(\cdot, S: N)$ ) we use a certain Riemannian metric, it is omitted from the notation introduced in the above definition, which is justified by the following

Proposition 8. The numbers $c(a, S: N)$ and $c(u, S: N)$ are independent of the choice of Riemannian metric $g \in \mathcal{G}_{g_{M} \oplus g_{0}}$. 
Proof. Choose $g^{\alpha}, g^{\beta} \in \mathcal{G}_{g_{M} \oplus g_{0}}$ and introduce auxiliary invariants $c\left(a, g^{\alpha}, S: N\right)$ and $c\left(u, g^{\beta}, S: N\right)$ defined via $g^{\alpha}$ and $g^{\beta}$. Recall that the isomorphism

$$
h_{\alpha \beta}: H_{*}\left(g^{\alpha}, S\right) \rightarrow H_{*}\left(g^{\beta}, S\right)
$$

is induced by the group homomorphism

$$
\begin{gathered}
h_{\sharp}: C F_{*}\left(S_{N}\right) \rightarrow C F_{*}\left(S_{N}\right), \\
h_{\sharp}\left(x^{\alpha}\right)=\sum_{x^{\beta}} n\left(x^{\alpha}, x^{\beta}\right) x^{\beta},
\end{gathered}
$$

where $n\left(x^{\alpha}, x^{\beta}\right)$ is the algebraic number of points in the zero-dimensional manifold $\mathcal{M}_{\left(g^{\alpha \beta}, S\right)}\left(x^{\alpha}, x^{\beta}\right)$ of solutions of equation (5) which connect $x^{\alpha}$ to $x^{\beta}$. We compute the difference $S\left(x^{\beta}\right)-S\left(x^{\alpha}\right)$ for every $x^{\beta}$ which appears in sum (15), i.e for such $x^{\beta}$ for which the set $\mathcal{M}_{\left(g^{\alpha \beta}, S\right)}\left(x^{\alpha}, x^{\beta}\right)$ is nonempty $\left(n\left(x^{\alpha}, x^{\beta}\right) \neq 0\right)$. For any $\gamma \in$ $\mathcal{M}_{\left(g^{\alpha \beta}, S\right)}$

$$
\begin{aligned}
S\left(x^{\beta}\right)-S\left(x^{\alpha}\right) & =\int_{-\infty}^{+\infty} \frac{d}{d t} S(\gamma) d t \\
& =\int_{-\infty}^{+\infty} d S(\gamma) \frac{d \gamma}{d t} d t \\
& =\int_{-\infty}^{+\infty}\left\langle\nabla^{g_{t}^{\alpha \beta}} S, \frac{d \gamma}{d t}\right\rangle_{g_{t}^{\alpha \beta}} d t \\
& =-\int_{-\infty}^{+\infty}\left|\frac{d \gamma}{d t}\right|_{g_{t}^{\alpha \beta}}^{2} d t \\
& \leq 0 .
\end{aligned}
$$

Here we used (5). Therefore $S\left(x^{\alpha}\right) \geq S\left(x^{\beta}\right)$, which implies that $h_{\alpha \beta}$ restricts to

$$
h_{\alpha \beta}: H_{*}^{\lambda}\left(g^{\alpha}, S\right) \rightarrow H_{*}^{\lambda}\left(g^{\beta}, S\right),
$$

and

$$
h_{\alpha \beta} \circ j_{*}^{\lambda}=j_{*}^{\lambda} \circ h_{\alpha \beta} .
$$

Assume that $T_{*}^{\alpha} a \in \operatorname{Im}\left(j_{*}^{\lambda}\right)$, where

$$
T_{*}^{\alpha}: H_{*}(N) \rightarrow H_{*}\left(g^{\alpha}, S\right) .
$$

Then, by (16), $h_{\alpha \beta} T_{*}^{\alpha} a \in \operatorname{Im}\left(j_{*}^{\lambda}\right)$. Since $h_{\alpha \beta} T_{*}^{\alpha}=T_{*}^{\beta}$, we have $T_{*}^{\beta} a \in \operatorname{Im}\left(j_{*}^{\lambda}\right)$ and hence

$$
c\left(a, g^{\alpha}, S: N\right) \geq c\left(a, g^{\beta}, S: N\right) .
$$

Since the above argument is valid for any $g^{\alpha}, g^{\beta}$, interchanging the role of $g^{\alpha}$ and $g^{\beta}$ we get the opposite inequality and hence

$$
c\left(a, g^{\alpha}, S: N\right)=c\left(a, g^{\beta}, S: N\right) .
$$

In order to consider $c(a, S: N)$ as an invariant of the Lagrangian submanifold generated by $S$ we prove that the transformations in Theorem 3 leave $c(a, \cdot: N)$ essentially unchanged. More precisely, we have the following 
Lemma 9. Let $S_{1}$ and $S_{2}$ be the two generating functions quadratic at infinity that generate the same Lagrangian submanifold $L \in T^{*} M$. Then there exists a constant (independent of $N$ ) $c_{0} \in \mathbb{R}$ such that for any $a \in H_{*}(N)$ and for any $u \in H^{*}(N)$

$$
c\left(a, S_{1}: N\right)=c\left(a, S_{2}: N\right)+c_{0}, c\left(u, S_{1}: N\right)=c\left(u, S_{2}: N\right)+c_{0} .
$$

Proof. By Theorem 3 and Proposition 8 it is enough to prove that with the suitable choice of metrics the Morse complex of $S$ does not change after the stabilization $S \leadsto$ $S \oplus Q$ and the transformation $S \sim S \circ \Phi+c_{0}$ with a fiber preserving diffeomorphism $\Phi$ and a constant $c_{0} \in \mathbb{R}$. The first statement follows immediately from

$$
C_{p}\left(S_{N}\right) \cong C_{p+\operatorname{index}(Q)}\left(S_{N} \oplus Q\right), \mathcal{M}_{S_{N}, g}(E) \cong \mathcal{M}_{S_{N} \oplus Q, g \oplus g_{0}}\left(E \times \mathbb{R}^{l}\right) .
$$

Assume now that $\Phi: E \rightarrow E$ is a fiber preserving diffeomorphism. If $S \in \mathcal{S}_{(E, Q)}$, then the transformation $S \leadsto S \circ \Phi$ does not change the Morse complex of $S$ with the suitable choice of metric, as the following argument shows. Let $g_{1}$ be a metric in $\mathcal{G}_{g_{M} \oplus g_{0}}$ and let $g_{2}:=\Phi_{*} g_{1}$ be a push-forward of $g_{1}$. Then

$$
\nabla^{g_{1}}\left(\Phi^{*} S\right)=\Phi^{*}\left(\nabla^{g_{2}} S\right)
$$

and hence if $\gamma: \mathbb{R} \rightarrow E$ is a solution of

then $\tilde{\gamma}:=\Phi \circ \gamma$ satisfies

$$
\frac{d \gamma}{d t}+\nabla^{g_{1}} S_{N}(\gamma)=0
$$

$$
\begin{aligned}
\frac{d \tilde{\gamma}}{d t}+\nabla^{g_{2}} S_{N}(\tilde{\gamma}) & =T \Phi\left(\frac{d \gamma}{d t}+(T \Phi)^{-1} \nabla^{g_{2}} S_{N}(\tilde{\gamma})\right) \\
& =T \Phi\left(\frac{d \gamma}{d t}+\nabla^{g_{1}} S_{N}(\gamma)\right) \\
& =0 .
\end{aligned}
$$

Therefore

$$
\mathcal{M}_{S_{N}, g_{1}}(E) \cong \mathcal{M}_{\Phi^{*} S_{N}, g_{2}}(E) .
$$

In particular, there exists a compact set $K_{0}$ such that all gradient trajectories (in metric $\Phi_{*} g_{1}$ ) which satisfy (14) remain in $K_{0}$. Note that for every negative gradient trajectory $\gamma$ starting at the point $x \in \operatorname{Crit}(S \circ \Phi)$ and leaving $K_{0}$ (i.e. not satisfying (14))

$$
\lim _{\tau \rightarrow \infty} S \circ \Phi(\gamma(\tau))=-\infty
$$

Hence there exists a relatively compact open set $U_{1} \supset K_{0}$ such that for any such $\gamma$ and any $\tau \in \mathbb{R}$ such that $\gamma(\tau) \notin U_{1}$

$$
S \circ \Phi(\gamma(\tau))>\min \{S \circ \Phi(x) \mid x \in \operatorname{Crit}(S \circ \Phi)\} .
$$

Choose a relatively compact open set $U_{2} \supset \bar{U}_{1}$ and define the metric $g_{3}$ by

$$
g_{3}= \begin{cases}\Phi_{*} g_{1} & \text { in } U_{1}, \\ g_{1} & \text { outside } \bar{U}_{2} .\end{cases}
$$

Recall that all gradient trajectories which satisfy (14) end at the critical point of $S \circ \Phi$. Since $S \circ \Phi$ decreases along negative gradient trajectories, it follows from (17) and the construction of a metric $g_{3}$ that all $g_{3}$-gradient trajectories of $S \circ \Phi$ satisfying (14) remain in $U_{1}$. Therefore

$$
\mathcal{M}_{S_{N}, g_{1}}(E) \cong \mathcal{M}_{\Phi^{*} S_{N}, g_{3}}(E)
$$


and

$$
C_{p}\left(S_{N}\right) \cong C_{p}\left(S_{N} \circ \Phi\right) .
$$

Note that, since $\nabla S=\nabla\left(S+c_{0}\right)$ for a constant $c_{0} \in \mathbb{R}$, Morse theory also applies to the functions in

$$
\mathcal{S}_{(E, Q)}+c_{0}:=\left\{S+c_{0} \mid S \in \mathcal{S}_{(E, Q)}\right\} .
$$

Hence $c\left(a, S+c_{0}: N\right)$ is well defined and, obviously,

$$
c\left(a, S+c_{0}: N\right)=c(a, S: N)+c_{0} .
$$

This finishes the proof.

The following theorem establishes the main properties of $c$ :

Theorem 10 (Compare with [20]). Let $N \subset M$ and $S_{N}$ be as before. Denote the conormal bundle of $N$ in $T^{*} M$ by $\nu^{*} N$. Let $L_{S} \subset T^{*} M$ be a Lagrangian submanifold generated by $S$.

1. $c(a, S: N)$ and $c(u, S: N)$ are the critical values of $S_{N}$.

2. For $a, b \in H_{*}(N)$ there exist points $x_{a}, x_{b} \in \nu^{*} N \cap L_{S}$ such that

$$
c(a, S: N)-c(b, S: N)=\int_{\gamma} \theta
$$

for any smooth path $\gamma$ in $L_{S}$ connecting $x_{a}$ to $x_{b}$.

3. The function $c(a, \cdot: N): S \mapsto c(a, S: N)$ from $\mathcal{S}_{(E, Q)}$ to $\mathbb{R}$ is $C^{0}$-continuous.

Hence, we can define $c(a, S: N)$ for any (not necessarily generic) $S$ by

$$
c(a, S: N):=\lim _{S_{n} \rightarrow S} c\left(a, S_{n}: N\right) .
$$

4. If $S_{i}: E_{i} \rightarrow \mathbb{R}, i=1,2$ are two generating functions quadratic at infinity and

$$
\begin{gathered}
S^{3}:=S^{1} \sharp S^{2}: E_{1} \oplus E_{2} \rightarrow \mathbb{R}, \\
S^{3}\left(\zeta_{1} \oplus \zeta_{2}\right)=S^{1}\left(\zeta_{1}\right)+S^{2}\left(\zeta_{2}\right),
\end{gathered}
$$

then

$$
c\left(u \cup v, S^{3}: N\right) \geq c\left(u, S^{1}: N\right)+c\left(v, S^{2}: N\right)
$$

for any $u, v \in H^{*}(N)$.

5. If $N$ is oriented, then

$$
c\left(\Gamma_{*}(a), S: N\right)=-c(a,-S: N)
$$

where $\Gamma_{*}$ is as in Section 3.1.

6. If $M$ is oriented and connected and $\mu \in H_{n}(M)$ and $1 \in H_{0}(M)$ are the generators of $H_{n}(M)$ and $H_{0}(M)$, then

$$
c(\mu, S: M) \geq c(1, S: M)
$$

and $c(\mu, S: M)=c(1, S: M)$ if and only if $S$ generates the zero section $o_{M}$. Statements 5. and 6. in non-orientable cases hold in $\mathbb{Z}_{2}$ coefficients. 
Proof. We give a proof of this theorem using the machinery of Morse homology, motivated by the ideas of $[14,13]$. For the proof of analogous theorem by methods of algebraic and differential topology we refer the reader to the original paper [20].

Since $S-Q$ has compact support, there are only finitely many critical points of $S$. In particular,

$$
H_{*}^{-\lambda}\left(S_{N}\right)=0 \text { and } H_{*}^{\lambda}\left(S_{N}\right)=H_{*}^{\infty}\left(S_{N}\right)
$$

for $\lambda$ large enough. Hence $c \neq \pm \infty$ and thus 1 . follows from the definition and the fact that

$$
j_{*}: H_{*}^{\lambda}\left(S_{N}\right) \rightarrow H_{*}^{\lambda+\varepsilon}\left(S_{N}\right)
$$

is an isomorphism if there are no critical values of $S$ in interval $[\lambda, \lambda+\varepsilon]$. Since $i_{S}\left(\operatorname{Crit}\left(S_{N}\right)\right)=\nu^{*} N \cap L_{S}, 2$. is a consequence of 1 . and the identity $i_{S}^{*} \theta=d\left(\left.S\right|_{\Sigma_{S}}\right)$.

Now we prove 3 . For Morse functions $S^{\alpha}, S^{\beta} \in \mathcal{S}_{(E, Q)}$ consider the connecting homotopy

$$
S^{\alpha \beta}=\rho S^{\beta}+(1-\rho) S^{\alpha} \in \mathcal{S}_{(E, Q)}
$$

where

$$
\rho: \mathbb{R} \rightarrow \mathbb{R}
$$

is a $C^{\infty}$ function such that

$$
\rho(\tau)= \begin{cases}1 & \text { for } \tau \geq 1 \\ 0 & \text { for } \tau \leq 0\end{cases}
$$

Then, we compute $S^{\beta}\left(x^{\beta}\right)-S^{\alpha}\left(x^{\alpha}\right)$ for a pair

$$
\left(x^{\alpha}, x^{\beta}\right) \in \operatorname{Crit}\left(S^{\alpha}\right) \times \operatorname{Crit}\left(S^{\beta}\right)
$$

connected by the trajectory $\gamma \in \mathcal{M}_{S^{\alpha \beta}, g}\left(x^{\alpha}, x^{\beta}\right)$. We obtain

$$
\begin{aligned}
S^{\beta}\left(x^{\beta}\right)-S^{\alpha}\left(x^{\alpha}\right) & =\int_{-\infty}^{+\infty} \frac{d}{d \tau} S^{\alpha \beta}(\gamma(\tau)) d \tau \\
& =\int_{-\infty}^{+\infty}\left[d S^{\alpha \beta}(\gamma(\tau)) \frac{d \gamma}{d \tau}+\rho^{\prime}(\tau)\left(S^{\beta}(\gamma(\tau))-S^{\alpha}(\gamma(\tau))\right)\right] d \tau \\
& \leq-\int_{-\infty}^{+\infty}\left\langle\nabla S^{\alpha \beta}(\gamma(\tau)), \frac{d \gamma}{d \tau}\right\rangle d \tau+\max \left(S^{\beta}-S^{\alpha}\right) \\
& \left.=-\int_{-\infty}^{+\infty}\left|\nabla S^{\alpha \beta}(\gamma(\tau))\right|^{2} d \tau+\max \left(S^{\beta}-S^{\alpha}\right) \text { (by } 4\right) \\
& \leq \max \left(S^{\beta}-S^{\alpha}\right) .
\end{aligned}
$$

The same estimate holds for any regular connecting homotopy sufficiently close to $S^{\alpha \beta}$. Hence, we have the commutative diagram

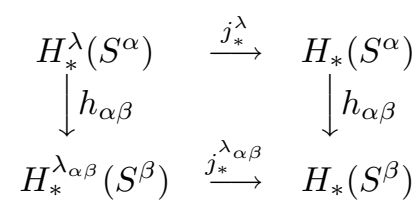

where $\lambda_{\alpha \beta}:=\lambda+\max \left(S^{\beta}-S^{\alpha}\right)$. Therefore, using the definition of $c$ we deduce

$$
c\left(a, S^{\beta}: N\right)-c\left(a, S^{\alpha}: N\right) \leq \max \left(S^{\beta}-S^{\alpha}\right) .
$$


Interchanging the role of $\alpha$ and $\beta$ we get

$$
c\left(a, S^{\alpha}: N\right)-C\left(a, S^{\beta}: N\right) \leq \max \left(S^{\alpha}-S^{\beta}\right)=-\min \left(S^{\beta}-S^{\alpha}\right)
$$

and hence

$$
c\left(a, S^{\beta}: N\right)-c\left(a, S^{\alpha}: N\right) \geq \min \left(S^{\beta}-S^{\alpha}\right) .
$$

Combining (18) and (19) we get

$$
\left|c\left(a, S^{\beta}: N\right)-c\left(a, S^{\alpha}: N\right)\right| \leq\left\|S^{\beta}-S^{\alpha}\right\|_{C^{0}} .
$$

To prove 4., we first assume that $S^{1}, S^{2}, S^{3}$ are (after stabilization) defined on $M \times \mathbb{R}^{m_{i}}$ for $i \in\{1,2,3\}$. Further, we extend $S^{1}$ and $S^{2}$ to whole $E:=M \times \mathbb{R}^{m_{1}+m_{2}}$ by

$$
S^{1}(q, \xi, \eta)=S^{1}(q, \xi)+\varepsilon|\eta|^{2}, S^{2}(q, \xi, \eta)=S^{2}(q, \eta)+\varepsilon|\xi|^{2},
$$

where $|\zeta|^{2}=\sum \zeta_{j}^{2}$ is the standard norm on $\mathbb{R}^{m}$ and $\varepsilon>0$. Since the stabilization $S \sim S \oplus Q$ does not change the value of $c(\cdot, S: N)$, we will prove 4 . for $S^{1}(q, \xi, \eta)$, $S^{2}(q, \xi, \eta)$ and

$$
S^{3}(q, \xi, \eta)=S^{1}(q, \xi)+S^{2}(q, \eta)\left(=S^{1}(q, \xi, \eta)+S^{2}(q, \xi, \eta)-\varepsilon\left(|\xi|^{2}+|\eta|^{2}\right)\right) .
$$

Note that, if

$$
T^{p_{1}}: H^{p_{1}}(N) \rightarrow H^{p_{1}+k_{1}}\left(S_{N}^{1}\right), T^{p_{2}}: H^{p_{2}}(N) \rightarrow H^{p_{2}+k_{2}}\left(S_{N}^{2}\right)
$$

and

$$
T^{p}: H^{p}(N) \rightarrow H^{p+k}\left(S_{N}^{3}\right), p=p_{1}+p_{2}, k=k_{1}+k_{2},
$$

then

$$
T^{p}(u \cup v)=T^{p_{1}}(u) \cup T^{p_{2}}(v) .
$$

Indeed, for $S^{i}=f \oplus Q^{i}(i \in\{1,2\})$, where $f$ is a Morse function on $N$, this equality is obvious since the stabilization $f \leadsto f \oplus Q$ does not change the Morse chain complex of $f$. Furthermore, if

$$
h^{\alpha \beta}: H^{p+k}\left(f \oplus Q^{i}\right) \rightarrow H^{p+k}\left(S_{N}^{i}\right)
$$

is the canonical isomorphism, then, by (7)

$$
h^{\alpha \beta}\left(T^{p_{1}}(u) \cup T^{p_{2}}(v)\right)=h^{\alpha \beta}\left(T^{p_{1}}(u)\right) \cup h^{\alpha \beta}\left(T^{p_{2}}(v)\right)
$$

and thus (21) follows.

Let $\gamma=\left(\gamma_{1}, \gamma_{2}, \gamma_{3}\right)$ be the solution of

$$
\left\{\begin{array}{l}
\frac{d \gamma_{i}}{d t}=-\nabla S^{i}\left(\gamma_{i}\right), \\
\lim _{t \rightarrow+\infty} \gamma_{1}(t)=x_{1}, \\
\lim _{t \rightarrow+\infty} \gamma_{2}(t)=x_{2}, \\
\lim _{t \rightarrow-\infty} \gamma_{3}(t)=x_{3}, \\
\gamma_{1}(0)=\gamma_{2}(0)=\gamma_{3}(0) .
\end{array}\right.
$$


Note that, since all $S_{i}$ are quadratic outside fixed compact set, we have $\gamma_{i} \subset K_{0}$ for some fixed compact set $K_{0}$. If we write $\gamma_{1}, \gamma_{2} \subset M \times \mathbb{R}^{m_{1}} \times \mathbb{R}^{m_{2}}$ as $\gamma_{i}(t)=$ $\left(x_{i}(t), \xi_{i}(t), \eta_{i}(t)\right)$ for $i \in\{1,2\}$, then

$$
\begin{aligned}
S^{1}\left(x_{1}\right)+S^{2}\left(x_{2}\right)-S^{3}\left(x_{3}\right) & =S^{1}\left(x_{1}\right)-S^{1}(\gamma(0))+S^{2}\left(x_{2}\right)-S^{2}(\gamma(0)) \\
& -S^{3}\left(x_{3}\right)+S^{3}(\gamma(0))+\varepsilon\left(\left|\xi_{1}(0)\right|^{2}+\left|\eta_{2}(0)\right|^{2}\right) \\
& \leq \varepsilon \max _{K_{0}}\left(|\xi|^{2}+|\eta|^{2}\right) \\
& =: \varepsilon_{1} .
\end{aligned}
$$

Here we used $S^{1}\left(\gamma_{1}(0)\right)+S^{2}\left(\gamma_{2}(0)\right)=S^{3}\left(\gamma_{3}(0)\right)+\varepsilon\left(\left|\xi_{1}(0)\right|^{2}+\left|\eta_{2}(0)\right|^{2}\right)$ (by (20), since $\left.\gamma_{1}(0)=\gamma_{2}(0)=\gamma_{3}(0)\right)$ and the fact that $S^{i}$ decreases along $\gamma_{i}$. Therefore,

$$
-S^{3}\left(x_{3}\right) \leq-S^{1}\left(x_{1}\right)-S^{2}\left(x_{2}\right)+\varepsilon_{1} .
$$

Assume that

$$
c\left(u, S^{1}: N\right)+c\left(v, S^{2}: N\right)>\lambda
$$

and choose $\lambda_{1}, \lambda_{2}$ such that $\lambda<\lambda_{1}+\lambda_{2}$ and $\lambda_{1}<c\left(u, S^{1}: N\right), \lambda_{2}<c\left(v, S^{2}: N\right)$. Choose the cocycles representing $U=T^{*} u \in H^{*}\left(S_{N}^{1}\right)$ and $V=T^{*} v \in H^{*}\left(S_{N}^{2}\right)$, denoted again by $U$ and $V$. We have

$$
U(x)=0, V(y)=0, \text { for } x \in \operatorname{Crit}^{\lambda_{1}}\left(S_{N}^{1}\right), y \in \operatorname{Crit}^{\lambda_{2}}\left(S_{N}^{2}\right) .
$$

Hence, the only nontrivial contributions to

$$
U \otimes V\rfloor \Psi\left(S_{N}^{1}, S_{N}^{2}, S_{N}^{3}\right):=\sum_{x_{1}, x_{2}, x_{3}} n\left(x_{1}, x_{2}, x_{3}\right)\left\langle U, x_{1}\right\rangle\left\langle V, x_{2}\right\rangle x_{3}
$$

come from the triples $\left(x_{1}, x_{2}, x_{3}\right)$ with

$$
-S^{3}\left(x_{3}\right) \leq-S^{1}\left(x_{1}\right)-S^{2}\left(x_{2}\right)+\varepsilon_{1}<-\lambda_{1}-\lambda_{2}+\varepsilon_{1} .
$$

Therefore

$$
U \otimes V\rfloor \Psi\left(S_{N}^{1}, S_{N}^{2}, S_{N}^{3}\right) \in C_{*}^{-\lambda_{1}-\lambda_{2}+\varepsilon_{1}}\left(-S_{N}^{3}\right) .
$$

By definition of $\Gamma$ (see Section 3.1), we have

$$
\Gamma(U \otimes V\rfloor \Psi)\left.\right|_{C_{*}^{\lambda_{1}+\lambda_{2}-\varepsilon_{1}}\left(S_{N}^{3}\right)} \equiv 0 .
$$

Therefore $j_{\lambda-\varepsilon_{1}}^{*}\left(T^{*} u \cup T^{*} v\right)=0$, i.e.

$$
\lambda-\varepsilon_{1} \leq c\left(u \cup v, S^{3}: N\right)
$$

for every $\lambda$ satisfying (22). Hence

$$
c\left(u \cup v, S^{3}: N\right)+\varepsilon_{1} \geq c\left(u, S^{1}: N\right)+c\left(v, S^{2}: N\right) .
$$

Letting $\varepsilon \rightarrow 0$ (and hence $\varepsilon_{1} \rightarrow 0$ ) we prove 4 .

For the proof of 5 . we consider the groups

$$
C_{*}^{(\lambda, \mu)}(S):=C_{*}^{\mu}(S) / C_{*}^{\lambda}(S)
$$

and

$$
C_{(\lambda, \mu)}^{*}(S):=\operatorname{Hom}\left(C_{*}^{(\lambda, \mu)}(S), R\right) .
$$

The boundary operator $\partial$ induces

$$
\partial_{p}^{(\lambda, \mu)}: C_{p}^{(\lambda, \mu)}(S) \rightarrow C_{p-1}^{(\lambda, \mu)}(S)
$$


and

$$
\delta_{(\lambda, \mu)}^{p}:=\operatorname{Hom}\left(\delta_{p}^{(\lambda, \mu)}\right): C_{(\lambda, \mu)}^{p}(S) \rightarrow C_{(\lambda, \mu)}^{p+1}(S) .
$$

Hence we can define the relative homology and cohomology groups

$$
\begin{gathered}
H_{p}^{(\lambda, \mu)}(S):=\operatorname{Ker}\left(\partial_{p}^{(\lambda, \mu)}\right) / \operatorname{Im}\left(\partial_{p}^{(\lambda, \mu)}\right), \\
H_{(\lambda, \mu)}^{p}(S):=\operatorname{Ker}\left(\delta_{(\lambda, \mu)}^{p}\right) / \operatorname{Im}\left(\delta_{(\lambda, \mu)}^{p-1}\right) .
\end{gathered}
$$

Note that, by definition of $\Gamma_{*}$,

$$
\Gamma_{*}: H_{*}^{(-\mu,-\lambda)}(S) \rightarrow H_{(\lambda, \mu)}^{*}(-S)
$$

with homology and cohomology groups defined with respect to the orientations $\sigma$ and $\tilde{\sigma}$ as in Section 3.1. The short exact sequences

$$
0 \rightarrow C_{*}^{(-\infty,-\lambda)}(S) \rightarrow C_{*}(S) \rightarrow C_{*}^{(-\lambda,+\infty)}(S) \rightarrow 0
$$

and

$$
0 \rightarrow C_{(\lambda,+\infty)}^{*}(S) \rightarrow C^{*}(S) \rightarrow C_{(-\infty, \lambda)}^{*}(S) \rightarrow 0
$$

generated by obvious inclusion maps give rise to the long exact sequences in homology and cohomology such that the diagram

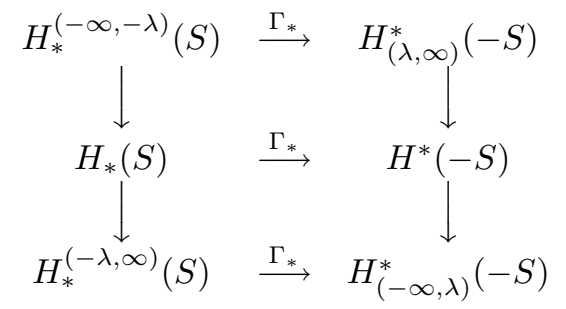

commutes. By exactness of vertical columns, $j_{\lambda}^{*} \Gamma_{*}\left(T_{*} a\right) \neq 0$ is equivalent to $T_{*} a \in$ $\operatorname{Im}\left(j_{*}^{-\lambda}\right)$. Therefore, if $\lambda<c\left(\Gamma_{*}(a),-S: N\right)$, then $-\lambda \geq c(a, S: N)$ and thus $\lambda \leq-c(a, S: N)$. This proves

$$
c\left(\Gamma_{*}(a),-S: N\right) \leq-c(a, S: N) .
$$

Conversely, if $\lambda<-c(a, S: N)$, then, by definition of $c, T^{*} a \in \operatorname{Im}\left(j_{*}^{-\lambda}\right)$. By exactness $j_{\lambda}^{*} \Gamma_{*}\left(T_{*}(a)\right)=0$ and thus $\lambda \leq c\left(\Gamma_{*}(a), S: N\right)$. That proves

$$
-c(a, S: N) \leq c\left(\Gamma_{*}(a), S: N\right) .
$$

This finishes the proof of 5 .

In order to prove 6 . we first extend the definition of cap product given in Section 3. Consider a point $z \in M$ and the fiber $\pi^{-1}(z) \subset E$. For $x \in C_{n+k}(S)$ and $y \in C_{k}(S)$ we define

$$
\mathcal{M}_{S, g}^{z}(x, y):=\left\{\gamma \in \mathcal{M}_{S, g}(x, y) \mid \gamma(0) \in \pi^{-1}(z)\right\} .
$$

Consider the evaluation map

$$
\begin{gathered}
E v: \mathcal{M}_{S, g}(x, y) \rightarrow M, \\
E v(\gamma)=\pi(\gamma(0)),
\end{gathered}
$$

For a regular value $z$ of $E v \mathcal{M}_{S, g}^{z}(x, y)$ is a smooth zero dimensional manifold. Since all bounded gradient trajectories defined by parameters from $\mathcal{S}_{(E, Q)} \times \mathcal{G}_{g_{M} \oplus g_{0}}$ remain in a fixed compact subset of $E$, the compactness of $\mathcal{M}_{S, g}^{z}(x, y)$ follows from 
the same arguments as in Section 3. Hence we can define $n_{z}(x, y)$ as the algebraic number of points in $\mathcal{M}_{S, g}^{z}(x, y)$ and the map

$$
\psi_{z}: C_{n+k}(S) \rightarrow C_{k}(S)
$$

as the linear extension of the map

$$
x \mapsto \sum_{\beta} n_{z}(x, y) y .
$$

The chain property of $\psi_{z}$ follows as in Section 3 and thus $\psi_{z}$ descends to the map in a homology, denoted again by

$$
\mu_{M} \cap \cdot: H_{n+k}(S) \rightarrow H_{k}(S) .
$$

Moreover, the following diagram commutes:

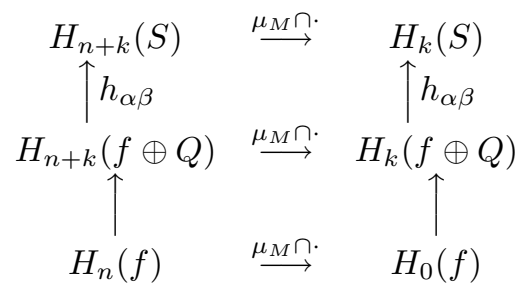

and all the arrows are the isomorphisms. For the lower rectangle this is again the consequence of the fact that the stabilization $f \leadsto f \oplus Q$ does not change the Morse chain complex of $f$; for the upper one this is the consequence of (13).

If $S$ does not generate $o_{M}$, then there exists a point $z_{0} \in M$ such that $d S(e) \neq 0$ for all $e \in \pi^{-1}\left(z_{0}\right)$. Since $S=Q$ outside a compact set, that means that

$$
\inf _{e \in \pi^{-1}\left(z_{0}\right)}|\nabla S|=2 \varepsilon_{0}>0 .
$$

Hence, there exist an $\varepsilon_{1}$-ball $U \subset M$ with the center at $z_{0}$ and a $C^{1}$ neighborhood $\mathcal{U} \subset \mathcal{S}_{(E, Q)}$ of $S$ such that

$$
\inf _{e \in \pi^{-1}(U)}\left|\nabla S_{0}\right|>\varepsilon_{0}>0
$$

for any $S_{0} \in \mathcal{U}$. Let $S_{l} \in \mathcal{U}$ be a sequence of generic functions such that

$$
C^{1}-\lim _{l \rightarrow \infty} S_{l}=S .
$$

Choose a generic $z_{l} \in U\left(:=B_{\varepsilon_{1}}\left(z_{0}\right)\right)$ which is $\frac{\varepsilon_{1}}{2}$-close to $z_{0}$. Let $\gamma_{l}$ be a negative gradient trajectory of $S_{l}$ with $\gamma_{l}(0) \in \pi^{-1}\left(z_{l}\right)$. By $(23)\left|\nabla S_{l}\left(\gamma_{l}(0)\right)\right|>\varepsilon_{0}>0$. Since

$$
\lim _{\tau \rightarrow \pm \infty} \nabla S_{l}\left(\gamma_{l}(\tau)\right)=0
$$

there exist $\tau_{l} \in \mathbb{R}$ such that

$$
\gamma_{l}\left(\tau_{l}\right) \notin \pi^{-1}(U)
$$


and thus

$$
\begin{aligned}
S_{l}\left(\gamma_{l}(+\infty)\right)-S_{l}\left(\gamma_{l}(-\infty)\right) & =\int_{-\infty}^{+\infty} \frac{d}{d \tau} S_{l}\left(\gamma_{l}(\tau)\right) d \tau \\
& =-\int_{-\infty}^{+\infty}\left|\nabla S_{l}\left(\gamma_{l}(\tau)\right)\right|^{2} d \tau \\
& \leq-\int_{0}^{+\tau_{l}}\left|\frac{d \gamma_{l}}{d \tau}\right|^{2} d \tau \\
& \leq-\left(\operatorname{dist}\left(z_{l}, M \backslash U\right)\right)^{2} \\
& \leq-\frac{\varepsilon_{1}^{2}}{4}=:-\varepsilon
\end{aligned}
$$

Hence from the non-degeneracy of cap action we obtain the isomorphism

$$
\mu_{M} \cap \cdot: H_{n+k}^{\lambda}\left(S_{l}\right) \rightarrow H_{k}^{\lambda-\varepsilon}\left(S_{l}\right) .
$$

Since $H_{0}(M)$ and $H_{n}(M)$ are generated by one generator, from the commutative diagram

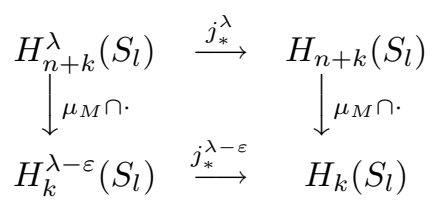

we conclude $c\left(\mu, S_{l}: M\right)-c\left(1, S_{l}: M\right)>\varepsilon$. Now 6 . follows by (24) and the continuity of $c(\mu, \cdot: M)-c(1, \cdot: M)$.

\section{ACKNOWLEDGMENTS}

I would like to thank Y.-G. Oh for valuable discussions during the preparation of this paper. This work is partially supported by Serbian National Foundation grant \#04M03.

\section{REFERENCES}

[1] M. Betz and R. Cohen. Graph moduli spaces and cohomology operations. Turkish J. Math., 18:23-41, 1994. MR 95i:58037

[2] M. Chaperon. Une idée du type "géodésiques brisées" pour les systèmes hamiltoniens. C. R. Acad. Sc. Paris, 298:293-296, 1984. MR 86f:58049

[3] M. Chaperon. An elementary proof of the Conley-Zehnder theorem in symplectic geometry. In Braaksma, Broer, and Takens, editors, Dynamical Systems and Bifurcations, volume 1125 of Springer Lecture Notes in Mathematics, pages 1-8, 1985. MR 87a:58062

[4] A. Floer. Morse theory for Lagrangian intersections. J. Diff. Geom., 28:513-517, 1988. MR 90f: 58058

[5] A. Floer. Cuplength estimates on Lagrangian intersections. Comm. Pure. Appl. Math., 42:335-356, 1989. MR 90g:58034

[6] A. Floer. Witten's complex and infinite dimensional Morse theory. J. Diff. Geom., 30:207221, 1989. MR 90d:58029

[7] K. Fukaya. Morse theory and topological field theory. To appear in Sugaku Expositions.

[8] K. Fukaya and Y.-G. Oh. Zero loop open strings in the cotangent bundle and Morse homotopy. Asian J. Math., 1: 96-180, 1997. CMP 98:04

[9] L. Hörmander. Fourier integral operators I. Acta Math., 127:79-183, 1971. MR 52:9299

[10] F. Laudenbach and J.-C. Sikorav. Persistence d'intersections avec la section nulle au conours d'une isotopie Hamiltonienne dans un fibre cotangent. Invent. Math., 82:349-357, 1985. MR 82c:58042 
[11] D. Milinković and Y.-G. Oh. Generating functions versus the action functional- stable Morse theory versus Floer theory. To appear in Proceedings of Workshop on Geometry, Topology and Dynamics, Montreal, 1995.

[12] J. Milnor. Lectures on the h-cobordism theorem. Math. Notes. Princeton Univ. Press, 1965. MR 32:8352

[13] Y.-G. Oh. Symplectic topology as the geometry of action functional II. Preprint.

[14] Y.-G. Oh. Symplectic topology as the geometry of action functional I. J. Diff. Geom., 46:499577, 1997. MR 99a:58032

[15] M. Schwarz. Morse Homology. Birkhäuser, Basel, 1993. MR 95a:58022

[16] J.-C. Sikorav. Problèmes d'intersections et de points fixes en géometrie Hamiltonienne. Comment. Math. Helv, 62:61-73, 1987. MR 88g:58067

[17] S. Smale. The generalized Poincaré conjecture in higher dimensions. Bull. Amer. Math. Soc., 66:373-375, 1960. MR 23:A2220

[18] D. Théret. Utilisation des fonctions generatrices en geometrie symplectique globale. $\mathrm{PhD}$ thesis, Université de Paris 7, 1995.

[19] L. Traynor. Symplectic homology via generating functions. GAFA, 4:718-784, 1994. MR 96a: 58049

[20] C. Viterbo. Symplectic topology as the geometry of generating functions. Math. Ann., 292:685-710, 1992. MR 93b:58058

[21] E. Witten. Supersymmetry and Morse theory. J. Diff. Geom., 17:661-692, $1982 . \quad$ MR 84b:58111

Department of Mathematics, University of Wisconsin-Madison, 480 Lincoln Drive, MADISON, WISCONSIN 53706

Current address: Department of Mathematics, University of California, Irvine, California 92697-3875

E-mail address: dmilinko@math.uci.edu 